\title{
Rastreamento de referência nas línguas Sakurabiat e Wayoro: uma análise contrastando cláusulas matrizes e subordinadas
}

\author{
Reference tracking in the Sakurabiat and Wayoro languages: an analysis of matrix \\ and subordinate clauses
}

\author{
Ana Vilacy GALUCIO* \\ Museu Paraense Emílio Goeldi (MPEG)
}

Antonia Fernanda NOGUEIRA ${ }^{* *}$

Universidade Federal do Pará (UFPA)

\begin{abstract}
RESUMO: A interrelação entre identificação de relações gramaticais, alinhamento morfossintático e rastreamento de referências é um campo de estudo ainda pouco investigado para línguas amazônicas. Os principais sistemas de identificação da relação entre sintagmas nominais argumentos e predicados são a ordem de constituintes, o caso nominal e a concordância verbal. Dentre esses sistemas, as línguas Tupi utilizam, em geral, uma combinação de ordem de constituintes e concordância/indexação verbal. Este estudo envolve línguas do ramo Tupari (Tupi), caracterizado por um sistema de alinhamento híbrido nominativoabsolutivo. Analisaremos dados das línguas Sakurabiat e Wayoro, aplicando a perspectiva tipológico-funcional (HASPELMATH, 2011; SHOPEN, 2007) para avançar na descrição do(s) sistema(s) de alinhamento, com foco especial na análise do rastreamento de referência na estrutura informacional. A análise de relações anafóricas e de controle entre cláusulas principais e subordinadas indica que os argumentos S/A funcionam como pivôs para o controle e identificação de referência.
\end{abstract}

PALAVRAS-CHAVE: Rastreamento de referência. Alinhamento morfossintático. Indexação verbal. Línguas Tupari. Família linguística Tupi.

ABSTRACT: The relationship between the identification of grammatical relations, morphosyntactic alignment and reference tracking is a field of study that is still underinvestigated for Amazonian languages. The main systems for identifying the relationship between noun phrase arguments and predicates are constituent order, nominal case and verbal agreement. Among these systems, Tupian languages generally use a combination of constituent order and verbal agreement/indexation. This study involves languages of the Tupari branch (Tupian family), characterized by a nominative-absolutive alignment system. We will analyze data from the Sakurabiat and Wayoro languages, applying the typological-functional perspective (HASPELMATH, 2011; SHOPEN, 2007) to advance the description of the

\footnotetext{
* Doutora em Lingüística pela University of Chicago (2001). Atualmente é pesquisadora titular do Museu Paraense Emilio Goeldi do Ministério de Ciencia Tecnologia e Inovação. E-mail: anavilacy@gmail.com

** Doutora em Linguística pelo programa de Linguística da Universidade de São Paulo. E-mail: fernandapakori@gmail.com
}

Revista Moara, n. 56, vol. 2, jan-jul 2021 ISSN: 0104-0944

Recebido em 30/04/2021

Aceito em 20/05/2021 
alignment system, with a special focus on the analysis of reference tracking in the information structure. The analysis of anaphoric and control relations between main and subordinate clauses indicates that the S/A arguments function as pivots for control and reference identification.

KEYWORDS: Reference tracking. Morphosyntactic alignment. Verbal indexing. Tuparian languages. Tupian language family.

\section{Introdução}

A inter-relação entre sistemas de identificação de relações gramaticais, alinhamento morfossintático e rastreamento de referências é um campo de estudo ainda pouco investigado para muitas línguas Amazônicas. Os principais sistemas de identificação da relação entre sintagmas nominais (SN) argumentos e seus predicados nas línguas do mundo são: ordem de constituintes, caso nominal e concordância verbal. Entre as línguas da família Tupi, existe grande variação interna quanto ao modo de identificar a relação entre o verbo e seus argumentos dentro da sentença. Dos três principais sistemas de identificação da relação entre sintagmas nominais argumentos e os predicados expressos nas línguas do mundo, as línguas Tupi utilizam, em geral, uma combinação do sistema de ordem de constituintes e do sistema de concordância ou indexação de argumentos no verbo, com destaque para o sistema de concordância ou indexação de argumentos. Entretanto, há diferenças nos subsistemas de codificação dos argumentos tanto entre ramos distintos da família quanto entre línguas do mesmo ramo (cf. Rodrigues e Cabral (2012), Birchall (2014), entre outros). Essa variação na forma de identificar as relações gramaticais $(\mathrm{S}, \mathrm{A}, \mathrm{O})$ e de expressar os argumentos no verbo resulta em distintos padrões de alinhamento morfossintático: absolutivo, nominativo, hierárquico, semântico etc. (JENSEN, 1998; RODRIGUES; CABRAL，2012; BIRCHALL, 2014, entre outros), descritos para as línguas Tupi.

As línguas da família Tupi exibem duas propriedades que estão diretamente relacionadas aos diferentes padrões de alinhamento morfossintático encontrados na família e às estratégias de identificação das relações gramaticais e rastreamento de referência. Essas propriedades são a ocorrência de duas séries de morfemas pessoais morfemas livres (pronomes) e presos (prefixos ou clíticos) - e a existência de um contraste entre formas pronominais identificadas como correferenciais e formas pronominais não-correferenciais (GALUCIO, 2014).

Revista Moara, n. 56, vol. 2, jan-jul 2021 ISSN: 0104-0944 
De modo geral, a indexação de argumentos nos verbos é associada à distribuição das diferentes séries de pronomes pessoais como uma das principais propriedades usadas para distinguir os argumentos e identificar relações gramaticais. Por exemplo, no ramo Tupi-Guarani, os morfemas presos são divididos em três ou quatro subconjuntos, distribuídos de acordo com o sistema de concordância e marcação de argumentos. Já as línguas dos ramos linguísticos falados em Rondônia (Arikém, Mondé, Ramarama, Tupari) compartilham um padrão absolutivo na indexação dos argumentos verbais em cláusulas matrizes, que se caracteriza por ter $\mathrm{S} / \mathrm{O}$ indexados no verbo com o mesmo grupo de prefixos pessoais.

Este estudo tem como foco duas línguas do ramo Tupari da família Tupí, que é composto pelas línguas Akuntsu, Wayoro, Makurap, Sakurabiat e Tupari. Todas as cinco línguas Tupari são faladas no atual estado de Rondônia, na Amazônia brasileira, especificamente nas Áreas Indígenas Rio Branco, Rio Guaporé e Rio Mequéns, conforme indicado no mapa na Figura 1. As cinco línguas Tupari encontram-se altamente ameaçadas, devido ao número reduzido de falantes e, no caso das línguas Sakurabiat, Wayoro e Akuntsú, também devido à interrupção da transmissão. De acordo com informações coletadas em 2017-2018 (GALUCIO, MOORE; VAN DER VOORT, 2018) e atualizadas para Sakurabiat em junho de 2021, Wayoro e Akuntsú possuem apenas 03 falantes cada; Sakurabiat apenas 11 falantes, Makurap cerca de 50 falantes e Tupari cerca de 300 a 340 falantes.

Figura 1: Mapa indicando as Terras Indígenas de Rondônia por Willem Doelman.

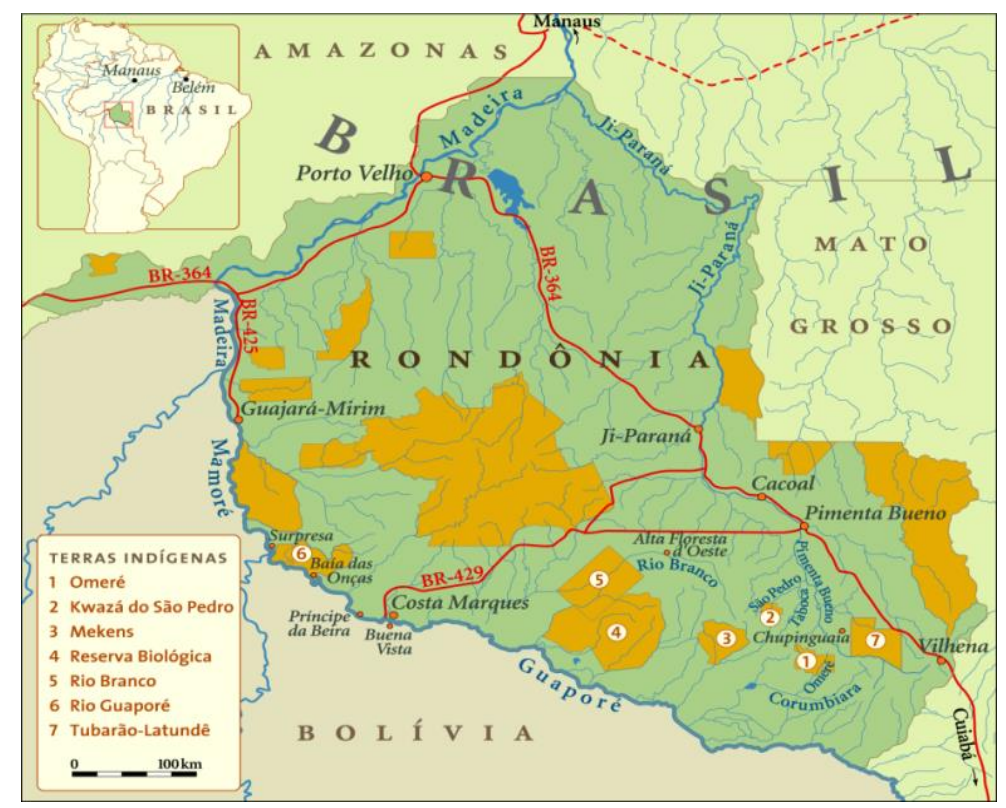

Revista Moara, n. 56, vol. 2, jan-jul 2021 ISSN: 0104-0944 
Fonte: van der Voort (2004)

Neste trabalho, analisaremos especificamente dados das línguas Sakurabiat e Wayoro, aplicando a perspectiva tipológico-funcional (HASPELMATH, 2011; SHOPEN, 2007) para avançar na descrição do(s) sistema(s) de alinhamento das línguas Tupari, com foco especialmente na análise do rastreamento de referência na estrutura informacional, conforme as definições delineadas em Matić, Van Gijn e van Valin Jr. (2014), incluindo a interação do sistema de rastreamento de referência com o sistema de indexação verbal em cláusulas subordinadas. Os resultados da análise de padrões sintáticos, como restrições de correferência entre argumentos de duas ou mais cláusulas, correferência de reflexivos e relações anafóricas e de controle entre cláusulas principais e subordinadas indicam que essas línguas apresentam uma prevalência de alinhamento nominativo, em que os argumentos S/A funcionam como pivôs para o controle de correferência e demais relações entre os argumentos de cláusulas distintas.

Os dados usados neste artigo são dados próprios, coletados pelas autoras, com falantes das línguas Sakurabiat e Wayoro, durante o curso de diversos anos de pesquisa sobre essas línguas. Em alguns casos, esses dados já foram publicados em trabalhos anteriores, conforme indicado, quando relevante. O acervo completo dos dados Sakurabiat encontra-se depositado no Acervo de Línguas Indígenas do Museu GoeldiALIM. O acervo da língua Wayoro encontra-se depositado parcialmente no ALIM/MPEG. A análise apresentada aqui se baseia tanto em dados provenientes de elicitação, direcionada para fenômenos linguísticos específicos, quanto em dados de fala natural, especialmente de narrativas. A apresentação dos dados segue as regras de apresentação de dados linguísticos e de convenções para representação interlinear da convenção de Leipzig $^{1}$ (COMRIE; HASPELMATH; BICKEL, 2008(2015)). Cada exemplo consta de três linhas: a representação fonêmica, a glossa morfema-pormorfema e a tradução livre para Português. As abreviaturas também seguem, sempre que possível, a lista de abreviaturas sugeridas na convenção de Leipzig. Uma lista completa das abreviaturas encontra-se ao final do artigo.

O restante deste artigo está organizado da seguinte maneira. Na seção 1, apresentamos uma breve caraterização tipológica das línguas do ramo Tupari da família Tupi, com ênfase no padrão de alinhamento morfossintático e de marcação de

\footnotetext{
${ }^{1}$ Leipzig glossing rules.
}

Revista Moara, n. 56, vol. 2, jan-jul 2021 ISSN: 0104-0944 
argumentos e sua relação com o sistema de identificação de relações gramaticais nessas línguas. A seção 2 introduz a noção de rastreamento de referência na estrutura informacional, analisando em detalhe duas estratégias principais encontradas nas línguas Sakurabiat e Wayoro: omissão de sujeitos correferentes e oposição entre dois índices de $3^{a}$ pessoa (correferencial e não-correferencial). Na seção 3 , apresenta-se uma hipótese preliminar para a origem desse sistema de rastreamento de referência, a partir da extensão de um sistema de marcação de argumentos e expressão de relações anafóricas no domínio local para o domínio de longa distância, em unidades maiores de discurso. Uma síntese da discussão e dos resultados da análise desenvolvida no artigo é apresentada na conclusão.

\section{Relações gramaticais e alinhamento morfossintático nas línguas Tupari}

Do ponto de vista tipológico, morfologicamente as línguas Tupari tendem a um perfil flexional/fusional, apresentando poucos morfemas por palavra. Com relação à sintaxe, essas línguas podem ser caracterizadas tipologicamente como línguas de núcleo final e núcleo marcado. Nas construções possessivas a ordem é possuidor - possuído, nos sintagmas verbais transitivos a ordem dos constituintes é objeto - verbo (OV). Não há marcação de caso morfológico indicando os papéis sintáticos centrais (A, S e O). Assim como em línguas dos outros ramos da família Tupi, a indicação das relações gramaticais entre os SN argumentos e os verbos é realizada através dos sistemas de ordem de constituintes e indexação de pessoa no verbo, especialmente no caso de argumentos pronominais. $\mathrm{O}$ argumento objeto indireto e os adjuntos são expressos em sintagmas posposicionais, nucleados por posposições definidas de acordo com a semântica do sintagma (dativo, locativo, ablativo). Exemplos (1a) e (1b) ilustram a ausência de caso morfológico e o papel exercido pela ordem de constituintes para a identificação de relações sintáticas na língua Sakurabiat.

\section{(1) Sakurabiat}

a. améko korĩpo sogo-a-t

cachorro/onça cobra morder-V.T-PASS

'O cachorro mordeu a cobra.'

Revista Moara, n. 56, vol. 2, jan-jul 2021 ISSN: 0104-0944 
b. korĩpo améko sogo-a-t

cobra cachorro/onça morder-V.T-PASS

'A cobra mordeu o cachorro.'

Com relação à marcação de argumentos nos verbos, Galucio e Nogueira (2018) descrevem um sistema de alinhamento híbrido do tipo nominativo-absolutivo para as línguas do ramo Tupari, semelhante ao descrito para algumas línguas Caribe e Jê (cf. Gildea e Castro Alves (2010, 2018)). Nas línguas Tupari, esse padrão de alinhamento é caracterizado, em sentenças matrizes, com base na indexação de argumentos nos verbos e na distribuição dos morfemas pronominais: pronomes livres indexam S/A, enquanto a série de prefixos ou clíticos de pessoa indexam S/O.

As línguas Tupari possuem as duas classes de morfemas de pessoa que ocorrem também em outros ramos da família Tupi: morfemas livres (pronomes) ${ }^{2}$ e morfemas presos, que são descritos para algumas línguas como prefixos pessoais e em outras como clíticos de pessoa ${ }^{3}$.

O padrão de alinhamento absolutivo $(\mathrm{S}=\mathrm{O})$ é expresso pelos índices de pessoa indexados ao verbo e coocorre com o padrão nominativo $(\mathrm{S}=\mathrm{A})$ manifesto na distribuição quasi-complementar entre pronomes e índices de pessoa. A mesma série de morfemas presos (índices de pessoa) indexados no verbo codifica o argumento absolutivo - sujeito de intransitivo $(\mathrm{S})$ e objeto $(\mathrm{O})$ - e a série de morfemas livres (pronomes) expressa o argumento nominativo - sujeitos transitivos (A) e intransitivos (S). O uso dos pronomes para indicar o argumento nominativo é obrigatório no caso de sujeitos transitivos de $1^{\mathrm{a}}$ e $2^{\mathrm{a}}$ pessoa e opcional para todos os sujeitos intransitivos e para sujeito transitivo de $3^{\mathrm{a}}$ pessoa, em algumas línguas. Por outro lado, o argumento O, quando pronominal, nunca é expresso por um morfema livre (pronome), sempre por um índice de pessoa indexado no verbo. Essa distribuição é apresentada de forma

\footnotetext{
2 Os pronomes reconstruídos para Proto-Tupari (GALUCIO; NOGUEIRA, 2018) para o singular são: 1P

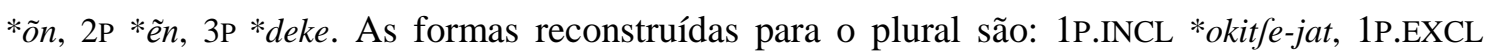
*otfe-jat, 2P*e(kitfe)-jat, 3P *ndeke-jat.

${ }^{3}$ Os morfemas pessoais presos reconstruídos para Proto-Tupari (GALUCIO; NOGUEIRA, 2018) para o singular são: $1 \mathrm{P} * o-, 2 \mathrm{P} * e-, 3 \mathrm{P} *$ tse- (sujeito de verbo intransitivo); $*_{i-} \sim *^{*}$ ts- (objeto de verbo transitivo). Para o plural, os morfemas presos reconstruídos são: 1P.INCL *ki-, 1P.EXCL *otfe-, 2P *e(ki)-, 3P *tse(sujeito de vero intransitivo); *i- $*^{*}$ tse- $\sim * d e(k e)$-jat (objeto de verbos transitivos)
}

Revista Moara, n. 56, vol. 2, jan-jul 2021 ISSN: 0104-0944 
esquematizada na tabela 1 abaixo.

Tabela 1: Alinhamento nominativo-absolutivo nas línguas Tupari

\begin{tabular}{rll}
\hline Função & Pronomes & Índices de pessoa \\
\hline A & Sim & \\
S & Sim (opcionalmente) & Sim \\
O & & Sim \\
\hline
\end{tabular}

Além dessa distribuição específica entre pronomes e índices de pessoa indexados nos verbos, as línguas Tupari apresentam outra propriedade interessante do ponto de vista tipológico-funcional. Apesar da mesma série de morfemas presos indexar sujeito intransitivo $(\mathrm{S})$ e objeto $(\mathrm{O})$, estes morfemas apresentam comportamento distinto dependendo da função gramatical do argumento indexado. Utilizando as definições de índices de pessoa propostas em Haspelmath (2013), as línguas Sakurabiat e Wayoro, e possivelmente também as outras línguas Tupari, fazem uma distinção entre crossíndices e pro-índices ${ }^{4}$. Os índices de sujeito (S) seriam classificados como cross-índices, uma vez que eles coocorrem com nomes e pronomes correferentes na função de sujeito. Já os índices de objeto $(\mathrm{O})$ seriam classificados como pro-índices, pois estão em distribuição complementar com sintagmas nominais e não podem ser conominados por pronomes livres na mesma posição, ou seja, os índices de objeto não coocorrem com nomes e pronomes na mesma função e referência (conominais) ${ }^{5}$. Essa distribuição é ilustrada nos exemplos (2a-b), para verbos transitivos e (3a-b) para verbos intransitivos, em Wayoro, e em (4a-b) e (5a-b), para verbos transitivos e intransitivos, respectivamente, em Sakurabiat.

(2) Wayoro

a. Pare eñ̃ aramĩra ombo-kwa-p ñ̃ txire

\footnotetext{
${ }^{4}$ Respectivamente, cross-indexes e pro-indexes, segundo Haspelmath (2013), correspondem a "Indexes with optional conominals: cross-indexes" e "Indexes with impossible conominal: proindexes". Ainda, conforme o autor, "Like free pronouns, pro-indexes occur in complementary distribution with full nominals, and they cannot be conominated by free pronouns either" (HASPELMATH, 2013, p. 219-220).

${ }^{5}$ Em uma abordagem baseada na gramática gerativa, a distribuição dos índices de argumentos nessas línguas pode ser descrita como um sistema misto, no qual os índices de sujeito nos verbos intransitivos (S) são analisados como indicadores de concordância, enquanto os índices de objeto nos verbos transitivos são analisados como pronomes cliticizados. Essa linha de análise é desenvolvida por Nogueira (2019) e Storto (2019, p. 73-77).
}

Revista Moara, n. 56, vol. 2, jan-jul 2021 ISSN: 0104-0944 
bom agora mulher bater-VZR-INF FUT nós.INCL

'Está bom! agora, nós vamos matar a mulher.'

b. Txi-ando-a-p na aramira txi=piki-a-t

1PL.INCL-?vingar-VZR-INF FUT mulher 1PL.INCL=acabar-V.T-NFUT

pare

bom

'Vamos nos vingar, a mulher está acabando com a gente, está bom!'

(3) Wayoro

a. txire txi-emẽto eri txi-apitngwar-a.

nós 1PL.INCL-idioma ABL 1PL.INCL-esquecer-V.T

'Nós esquecemos do nosso idioma.'

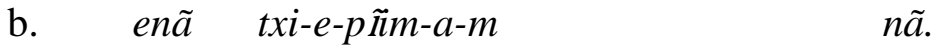

agora 1PL.INCL-MED-escuridão-V.T-INF FUT

'Agora, nós vamos anoitecer, [disse o dono da noite, uuy poot].'

(4) Sakurabiat

a. $o-s o-a-t \quad \tilde{e} t$

1SG-ver-V.T-PASS você

'Você me viu.'

b. ek obaat mõt-kwa o-koop

casa muito fazer-PL.EV 1SG-AUX.mover.IPFV.NPASS

'Eu estou construindo muitas casas.'

(5) Sakurabiat

a. $e-e r-a-t \quad(\tilde{e} t) \quad e n \tilde{\imath}=e s e$

2SG-dormir-V.T-PASS (você) rede $=$ LOC

'Você dormiu na rede.'

b. améko se-et-a i-toop

cachorro/onça 3C-dormir-VT 3SG-AUX.deitado.IPFV.NPASS

'O cachorro está dormindo.'

No caso de argumentos de terceira pessoa, há uma distinção entre duas formas pronominais, identificadas como correferencial e não-correferencial. $\mathrm{O}$ índice de $3^{\mathrm{a}}$ Revista Moara, n. 56, vol. 2, jan-jul 2021 ISSN: 0104-0944 
pessoa não-correferencial é $i$-/s- ${ }^{6}$ em Sakurabiat e $y-/ d j-1 \varnothing^{7}$ em Wayoro. Em sentenças matrizes, o prefixo não-correferencial é usado para indexar o objeto dos verbos transitivos. Enquanto o prefixo correferencial é usado para indexar o sujeito de verbos intransitivos e também o objeto reflexivo de um verbo transitivo. Além de marcar objeto (O) e sujeito intransitivo (S), os índices de pessoa também ocorrem com nomes, indexando o possuidor, em sintagmas nominais possessivos. Essa distribuição é ilustrada nos exemplos (6a-e) para Wayoro e (7a-f) para Sakurabiat.

Os exemplos (6a-b) e (7a-b) mostram que o índice de sujeito nos verbos intransitivos, o prefixo correferencial te- ' $3 C$ ' de Wayoro e se- ' $3 C$ ' de Sakurabiat, pode coocorrer com um sintagma nominal correferente na mesma função. A agramaticalidade de $(7 \mathrm{c})$ resulta do uso do índice de objeto, ou seja, o prefixo não-correferencial $i$ - ' 3 ', para indexar o sujeito intransitivo. Da mesma forma, a leitura indicada como agramatical em (6b) mostra que o uso do índice correferencial requer uma relação de correferência. Nas sentenças gramaticais (6c) e (7d), o prefixo não-correferencial $d j$ - ' 3 ' de Wayoro e $i$ - '3' de Sakurabiat ocorre indexando o objeto. Já os exemplos (6d) e (7e) mostram que o prefixo de $3^{\mathrm{a}}$ pessoa correferencial é usado na função de objeto, quando este é reflexivo, ou seja, quando seu referente é correferente com o sujeito da sentença ou quando o possuidor do objeto é correferente com o sujeito da sentença, em (6e) e (7f).

(6) Wayoro
a. mbogop te-apokay-a
criança $\quad 3 \mathrm{C}-$ sair-V.T
'A criança saiu.'
$\begin{array}{llll}\text { b. Enã } & \text { k-e-rom } & \text { piakarat } & \text { te-ukar-a-t } \\ \text { agora } & \text { ?-COP-NEG } & \text { galo/galinha } & \text { 3C-chorar-V.T-NFUT }\end{array}$
'De madrugada, o galo ${ }_{i}$ cantava $_{i} / *_{j}$ (Lit. 'Não é agora, o galo chorava')'
c. ndeke dj=ipit-kw-a-t
ele $\quad 3=$ jogar-VZR-V.T-NFUT

\footnotetext{
${ }^{6}$ Alomorfes condicionados fonologicamente. O alomorfe $i$ - ocorre antes de raízes iniciadas em consoante, e o alomorfe $s$ - antes de raízes iniciadas em vogal (cf. GALUCIO, 2014, p. 377).

${ }^{7}$ Alomorfes condicionados fonologicamente. Os alomorfes $y$ - $[n]$ e $d j$ - [dz] são usados diante de raízes iniciadas por vogais nasais e orais, respectivamente, e o alomorfe $\varnothing$ com raízes iniciadas por consoantes (cf. NOGUEIRA, 2019, p. 16).
}

Revista Moara, n. 56, vol. 2, jan-jul 2021 ISSN: 0104-0944 
'Ele jogou algo.'

d. Pororoka te-e-puru-ga-t

Pororoka 3C-MED-furar-VZR-NFUT

'Pororoka se furou (com flecha).'

e. Mariazinha te-upipe top-a-t

Mariazinha 3C-porto ver-V.T-NFUT

'Mariazinha ${ }_{i}$ viu o porto dela ${ }_{i /{ }^{*} j}$.

(7) Sakurabiat

a. améko se-kwear-a-t

cachorro/onça 3C-correr-V.T-PASS

'O cachorro correu.'

b. se-kwear-a-t

3C-correr-V.T-PASS

'Isto/ele/ela correu.'

c. *i-kwearat

d. (sete) i-so-a-t

ele/ela/isto 3SG-ver-V.T-PASS

'ele/ela/isto ${ }_{i}$ viu ele/ela/isto ${ }_{j}$.

e. se-so-a-t sete espelho-bo

3C-ver-V.T-PASS ele/ela/isto espelho-DAT

'Ele se viu no espelho.'

f. $\quad \tilde{a} s i_{i} \quad \operatorname{se}_{i}$-anüp tap sereka nããt

mãe 3C-cabeça cabelo cortar COP

'Minha mãe ${ }_{i}$ está cortando seu $_{i}$ próprio cabelo.'

É relevante mencionar que o contraste entre exemplos (6c) e (7d) de um lado e (6b) e (7c; 7e), por outro, ilustra um fenômeno muito conhecido da literatura que faz referência a um dos princípios centrais da teoria da regência e ligação (CHOMSKY, 1981) e que tem sido documentado como operante em várias línguas do mundo: o princípio B da teoria da ligação que se refere especificamente às restrições sintáticas relativas à atribuição de referência para os constituintes nominais em um determinado domínio sintático; ou de modo específico, trata das possibilidades de correferência entre

Revista Moara, n. 56, vol. 2, jan-jul 2021 ISSN: 0104-0944 
pronomes e seus antecedentes (ASUDEH; DALRYMPLE, 2006, p.23). Os exemplos de Wayoro e Sakurabiat mostram que o uso do pronome correferencial na posição de objeto só é licenciado quando o referente da expressão é correferente com o sujeito da sentença, ou seja, funciona como uma anáfora no domínio da sentença ${ }^{8}$.

$\mathrm{Na}$ próxima seção, discutiremos as estratégias utilizadas para o controle e rastreamento de identificação dos referentes em unidades maiores de discurso, seja em construções de coordenação, seja em construções de subordinação. Retomaremos também a discussão sobre o domínio da ligação e do controle de referência, mencionados acima, ao analisarmos o uso dos dois índices de $3^{a}$ pessoa (correferencial e não-correferencial) para indicar a referência dos participantes referidos nas expressões linguísticas. Como veremos a seguir, essa distinção está diretamente relacionada às estratégias de rastreamento da referência em unidades maiores do discurso, operando nas línguas analisadas.

\section{Rastreamento e controle de referência em sentenças complexas}

A noção de rastreamento de referência discutida aqui se refere à capacidade dos interlocutores do discurso de determinarem de forma inequívoca os referentes de uma dada expressão linguística (MATIĆ; VAN GIJN; VAN VALIN JR., 2014, p. 2) ${ }^{9}$. Nesse sentido, o rastreamento da referência em determinada expressão alcança sucesso se ambos interlocutores chegarem à mesma identificação dos referentes, ou seja, associarem o mesmo valor de referentes para uma dada expressão. Matić, van Gijn e van Valin Jr. (ibid., p.19-20) definem uma tipologia dos sistemas de rastreamento de referência em sentenças complexas baseada em quatro parâmetros: a estratégia de codificação dos argumentos, ou seja, a estratégia formal para marcar identidade e diferença na identidade dos referentes; o locus da marcação dessa identidade/diferença na identificação dos referentes, o domínio relevante para a interpretação da referência (local ou de longa distância); e a natureza (semântica, sintática ou pragmática) do controlador e do pivot nas relações de identidade/diferença de referência.

\footnotetext{
${ }^{8}$ A ocorrência de prefixos cognatos e com distribuição e função semelhante é descrita para as outras línguas do ramo Tupari (cf. SINGERMAN, 2018; ALVES, 2004; ARAGON, 2014).

${ }^{9}$ No original, "Reference tracking, refers to the capability of the interlocutors to unequivocally determine the referent(s) of a linguistic expression" (MATIĆ; VAN GIJN; VAN VALIN JR., 2014, p. 2).
}

Revista Moara, n. 56, vol. 2, jan-jul 2021 ISSN: 0104-0944 
Diversos tipos de estratégias são usados nas línguas do mundo para garantir o correto rastreamento da referência intercláusulas, envolvendo esses quatro parâmetros. Nesta seção, trataremos de duas estratégias morfossintáticas empregadas pelas línguas da família Tupari: a omissão de referentes idênticos e o uso contrastivo de duas formas de índices de $3^{a}$ pessoa (correferencial e não-correferencial). Com relação à forma de codificação, ambas estratégias podem ser classificadas como reducionistas, uma vez que a primeira envolve o apagamento do sujeito sob correferência e a segunda envolve o uso de uma forma de pronominalização. Nas duas línguas aqui analisadas, nas relações intercláusulas, embora se mantenha o sistema de alinhamento absolutivo de marcação dos argumentos nos verbos, via indexação dos índices de pessoa, descrito na seção 1 , observa-se uma prevalência de alinhamento nominativo a nível sintático, uma vez que os argumentos S/A funcionam como controladores do rastreamento de referência, de modo geral, tanto em sentenças coordenadas quanto em subordinadas. Nas duas línguas, a identidade ou a diferença de referência é geralmente indicada no $2^{\circ}$ membro de uma construção coordenada ou na cláusula dependente, no caso de construções subordinadas (cf. descrito por Galucio (2014) ${ }^{10}$, para Sakurabiat).

\subsection{Apagamento de sujeitos correferentes}

Como visto na seção 1 , em sentenças simples, os sujeitos de verbos transitivos são expressos por nominais ou pronomes, e não por índices de pessoa indexados ao verbo. Porém, exceto para os sujeitos de $3^{\mathrm{a}}$ pessoa, eles devem ser obrigatoriamente expressos. No caso de sentenças complexas, a identidade dos referentes pode afetar a marcação do sujeito, uma vez que sujeitos correferentes podem ser omitidos. Os exemplos (8) e (9) ilustram essa estratégia, para Sakurabiat e Wayoro, respectivamente.

(8) Sakurabiat (GALUCIO, 2014, p. 381)

$\begin{array}{llll}\text { Sakurap } & \text { aose } & \text { so- } a-t & i \text {-sek-a } \\ \text { macaco } & \text { homem } & \text { ver-VT-PASS } & \text { 3sG-agarrar-V.T }\end{array}$

'O homem viu o macaco e o agarrou.'

(9) Wayoro
Kap-ere,
kap-ere,
ndeke, petopngu poot,

\footnotetext{
${ }^{10}$ Neste trabalho, Galucio (2014) apresenta uma análise detalhada do sistema de rastreamento e controle de referência para a língua Sakurabiat.
}

Revista Moara, n. 56, vol. 2, jan-jul 2021 ISSN: 0104-0944 
PROFORMA-em PROFORMA-em ele raposa velho

te-tet-a, agopokap mẽ, emĩtok- $a, \quad$ kuy poan $\tilde{e}-a$

3C-ir-VT fogo/lenha TOP juntar-V.T terra amontoar-V.T

$y=$ angorog $-a$.

$3=$ torrar $-\mathrm{V} \cdot \mathrm{T}$

'Então, ele, Petop ngu, foi, juntou a lenha, amontoou a terra e a torrou.'

Essa estratégia reducionista envolvendo o apagamento de elementos referenciais normalmente exigidos nas sentenças independentes é comumente usada nas línguas do mundo para indicar a identidade de referentes, especialmente em sentenças coordenadas e em sentenças adverbiais (MATIĆ; VAN GIJN; VAN VALIN JR., 2014). Em Sakurabiat e em Wayoro, a estratégia de omissão de sujeitos correferentes ocorre também em sentenças subordinadas completivas, como ilustrado no exemplo (10) para a língua Wayoro. Como descrito por Nogueira (2019, p. 116), nesta sentença, o verbo transitivo ngwĩringã 'tecer/rolar' ocorre como núcleo do complemento do verbo ngwaynga 'começar'. O sujeito da sentença subordinada é correferente ao sujeito da sentença e controlado por este.

(10) Wayoro

$\begin{array}{lllll}\text { Ndeke } \text { ororo } & \text { ngwining- } \tilde{a}-m^{11} & \text { ngwayng-a-p } & n \tilde{a} \\ \text { Ele } & \text { algodão } & \text { tecer/rolar-V.T-INF } & \text { começar-V.T-INF } & \text { FUT }\end{array}$

'Ela vai começar a tecer linha de algodão.'

Em sentenças em que a identidade dos referentes não é idêntica, observa-se a estratégia de preservação na codificação, ou seja, os sujeitos não-correferenciais não são omitidos, como ilustrado pelos exemplos (11) e (12) abaixo. Porém, em unidades maiores de discurso, o domínio relevante para a interpretação da referência parece ser de longa distância, como veremos na próxima seção.

(11) Sakurabiat (GALUCIO, 2014, p. 381)

$\begin{array}{lllll}\text { aose } & \text { sakurap } & \text { so- } a-t & \text { te } & \text { sakurap } \\ \text { homem } & \text { macaco } & \text { ver-V.T-PASS } & \text { FOC } & \text { macaco }\end{array}$

\footnotetext{
${ }^{11}$ Os alomorfes do infinitivo são [m] após vogal nasal e [p] após vogal oral, conforme processo de neutralização entre consoantes oclusivas e consoantes nasais existente em Wayoro (cf. NOGUEIRA, 2011, p. 59-60).
}

Revista Moara, n. 56, vol. 2, jan-jul 2021 ISSN: 0104-0944 
$i$-sek-a

3SG-agarrar-V.T

'O homem viu o macaco e o macaco o agarrou.'

(12) Wayoro

on ororo emõ ngwĩring-ã ,

1SG algodão ENF tecer-V.T

'Eu costuro algodão mesmo.'

tipapnãn, Txuirika, ororo ngwĩring-ã

sogra Txuirika algodão tecer-V.T

'A sogra, Txuirika, costura algodão.'

aramĩa nõ-ian ororo ngwĩing-om

mulher outro-PL algodão tecer-NEG

'As outras mulheres não costuram algodão.'

\subsection{Oposição entre índices de $3^{a}$ pessoa correferencial e não-correferencial como estratégia de rastreamento e controle de referência}

A distinção entre formas pronominais correferenciais e não-correferenciais é uma característica comum a várias línguas da família Tupí, sendo que para a maioria das línguas, essa distinção é restrita à terceira pessoa. Embora a estrutura desse sistema possa variar entre as diferentes línguas da família, a distinção entre índices de pessoa correferenciais e não-correferenciais é geralmente empregada com verbos e nomes possessivos, para indicar identidade ou diferença na identidade de referentes, no domínio local, com relação ao sujeito da sentença (GALUCIO, 2014).

Essa distinção entre índices de pessoa correferenciais e não-correferenciais é relevante tanto no domínio local, quanto para relações de identidade/diferença de referência de longa distância e ao nível do discurso, uma vez que tanto os padrões de marcação de argumentos quanto à distinção entre índices de pessoa correferenciais e não-correferenciais estão interligados com a função de rastrear referentes no discurso.

Na seção 1, exemplificamos essa distinção entre os dois índices de $3^{\mathrm{a}}$ pessoa (correferencial e não-correferencial), no domínio local para as línguas Wayoro e Sakurabiat. Nesta seção, trataremos da extensão desse sistema para funcionar como 
estratégia de rastreamento e controle de referência em sentenças complexas.

Ao lado da estratégia tipologicamente comum nas línguas do mundo de omitir sujeitos idênticos nas relações interclausais, as línguas Tupari, assim como outras línguas Tupi, também empregam a distinção entre índices de $3^{\mathrm{a}}$ pessoa correferenciais e não-correferenciais para indicar identidades ou diferenças entre sujeitos de orações complexas $^{12}$. No caso das línguas em análise, devido à distribuição específica da indexação de argumentos, como descrito na seção 1, essa estratégia se aplica apenas a verbos intransitivos, em Wayoro, e a verbos intransitivos e auxiliares posicionais, em Sakurabiat, uma vez que essas são as únicas categorias lexicais que ocorrem com índices de sujeito.

Como descrito em Galucio (2014), em Sakurabiat, o índice de $3^{\mathrm{a}}$ pessoa nãocorreferencial é usado quando os sujeitos de duas (ou mais) orações relacionadas não são idênticos, enquanto o índice de $3^{a}$ pessoa correferencial é empregado para sujeitos idênticos em uma cadeia de cláusulas. Essa estratégia é exemplificada nos exemplos (13a-c) abaixo, para o domínio da sentença. Em (13a-b), o contraste entre esses dois índices de $3^{\text {a }}$ pessoa ocorre apenas na codificação do argumento nos auxiliares, enquanto em (13c) ocorre também no verbo intransitivo.

(13) Sakurabiat (GALUCIO, 2014)

a. améko aose sogo-a-t se-aot-a-ra

cachorro/onça homem morder-V.T-PASS 3C-sair-V.T-REP

se-seso-a

3C-AUX.andar-IPFV.PASS

'O cachorro mordeu o homem $_{j}$, quando ele ${ }_{i}$ ia saindo.'

b. améko aose sogo-a-t se-aot-a-ra

cachorro/onça homem morder-V.T-PASS 3C-sair-V.T-REP

i-seso- $a$

3SG-AUX.andar-IPFV.PASS

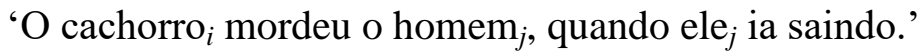

c. se-i-a te ikãw i-et-a i-to-a

\footnotetext{
${ }^{12}$ Uma análise desse sistema sob uma perspectiva tipológico-funcional para a família Tupi é tema de outro artigo, em preparação.
}

Revista Moara, n. 56, vol. 2, jan-jul 2021 ISSN: 0104-0944 
3C-vir-V.T FOC DEM 3SG-dormir-V.T 3SG-AUX.deitado-IPFV.PASS

$p e=o-m \tilde{e} t$.

OBL=1 SG-marido

'Ele $e_{j}$ veio na hora em que ele estava dormindo, meu marido $_{i}$ '

O trecho em (14a-e) abaixo, retirado de uma narrativa da língua Sakurabiat, ilustra tanto o uso da distinção entre os índices de $3^{\mathrm{a}}$ pessoa correferencial e nãocorreferencial, quanto a omissão de referentes idênticos, como estratégias de rastreamento e controle de referência, em uma unidade maior de discurso. A sentença (14a) introduz a narrativa e apresenta o primeiro participante, popoba 'coruja', que é o sujeito da primeira sentença. Neste caso, observa-se o índice de $3^{a}$ pessoa correferencial no verbo intransitivo, como previsto pelo sistema de marcação de argumentos (cf. Seção 1). Em (14b), ocorre a inclusão de um novo participante, o rapaz que imita a coruja. Este segundo participante é introduzido na forma de pronome (sete 'ele/ela') e na função de sujeito da segunda sentença (14b). O apagamento do sujeito em (14c) indica a correferência com o sujeito de (14b), o rapaz que é referido apenas pelo pronome de $3^{\mathrm{a}}$ pessoa (cf. seção 2.1 acima). Já em (14d), embora ocorra uma mudança na identidade do sujeito em relação à sentença anterior, observa-se também a omissão do sujeito do verbo transitivo que é correferente com o sujeito de (14a). Por outro lado, o uso do índice de $3^{\mathrm{a}}$ pessoa não-correferencial no verbo da cláusula subordinada em (14d) indica que os sujeitos da sentença matriz e da subordinada não são correferentes.

(14) Sakurabiat

\begin{tabular}{|c|c|c|c|c|c|}
\hline a. & $\begin{array}{l}\text { Popoba } \\
\text { coruja }\end{array}$ & $\begin{array}{l}\text { se-e-erek-kwa } \\
\text { 3C-INTRVZ-fala-TR.PI }\end{array}$ & EV & $\begin{array}{l}n \tilde{a} \tilde{a} t \\
\mathrm{COP}\end{array}$ & $\begin{array}{l}j \tilde{e} \\
\text { AUX.sentado.IPFV.PASS }\end{array}$ \\
\hline & 'A coruja estav & a cantando.' & & & \\
\hline & $k a a t=\tilde{e p}$ & $k w a g a t-k w a$ & sete & & \\
\hline & isto $=$ realmente & imitar-PL.EV & ele/ela & & \\
\hline & 'Ele (o jovem) & a imitou, a imitou.' & & & \\
\hline & $k a a t=\tilde{e p}$ & et & pro & & \\
\hline & isto $=$ realmente & dormir & pro & & \\
\hline & 'Então (ele) dor & rmiu.' & & & \\
\hline & pagop-taup & ese-jar-a- $t$ & & (pro) & \\
\hline
\end{tabular}

Revista Moara, n. 56, vol. 2, jan-jul 2021 ISSN: 0104-0944 


$\begin{array}{ll}\text { jovem-macho } & \text { SOC-sair-V.T-PASS (sujeito) } \\ i \text {-et- } a & i \text {-to- } a \\ \text { 3SG-dormir-V.T } & \text { 3SG-AUX.deitado-IPFV.PASS }\end{array}$

'Ela carregou o jovem quando ele estava dormindo.'

\begin{tabular}{|c|c|c|c|c|c|}
\hline \multirow[t]{2}{*}{ e. } & i-poetop & $n \tilde{e}-k w a$ & sasa & $e b a$ & sete \\
\hline & 3SG-conhecimento & COP-? & primeiro & EVID & ele/ela \\
\hline
\end{tabular}

Em Wayoro também se observa o uso do índice de $3^{\mathrm{a}}$ pessoa correferencial para indicar a identidade de referência entre sujeitos, em sentenças complexas e unidades maiores do discurso, como ilustrado no exemplo (15), abaixo. Assim, como no exemplo de Sakurabiat em (14), a sentença (15) mostra as duas estratégias de indicar sujeitos correferentes, em Wayoro. A sentença complexa em (15) contém uma oração coordenada, composta por uma sentença intransitiva e uma transitiva, e uma oração relativa (ou construção de foco do objeto ${ }^{13}$ ) como complemento da transitiva. $\mathrm{O}$ apagamento do sujeito no verbo transitivo ngura 'tirar' indica correferência com o sujeito do verbo intransitivo tera 'ir'. Por outro lado, observa-se o índice de $3^{\text {a }}$ pessoa correferencial tanto no verbo da sentença matriz (tera 'ir'), conforme descrito na seção 1, quanto no verbo da sentença relativa (pay 'achar') que funciona como modificador do objeto do verbo transitivo. O uso do índice de pessoa correferencial no verbo da oração relativa indica que o sujeito da relativa é correferente com o sujeito da sentença matriz.

(15) Wayoro

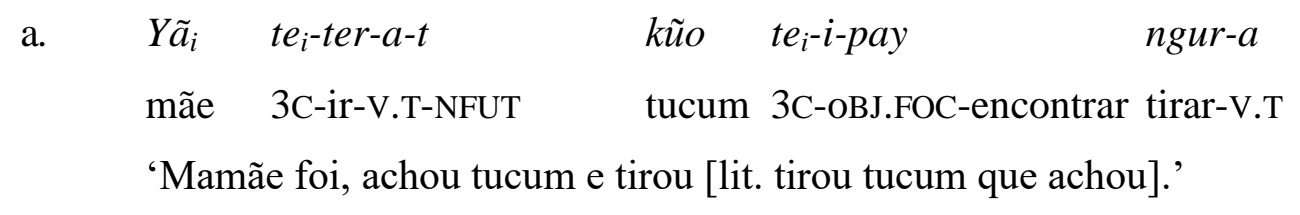

Portanto, em ambas línguas analisadas neste trabalho, o índice de $3^{\mathrm{a}}$ pessoa correferencial é usado sempre como uma anáfora indexado a um sujeito no domínio sintático apropriado, porém existe diferença na liberdade da escolha referencial que possibilita o uso desse índice de acordo com a língua. Em Sakurabiat, nas sentenças complexas, envolvendo relações de subordinação, não parece haver restrição de uso dos índices de $3^{\mathrm{a}}$ pessoa correferencial e não-correferencial. $\mathrm{O}$ índice não-correferencial é

${ }^{13}$ Cf. Galucio e Nogueira (2018).

Revista Moara, n. 56, vol. 2, jan-jul 2021 ISSN: 0104-0944 
usado tanto com verbos intransitivos quanto auxiliares para indicar que os sujeitos das duas orações relacionadas não são idênticos, enquanto o índice correferencial é usado para indicar sujeitos idênticos, independente do verbo da matriz.

Embora essa distinção entre os índices de $3^{\text {a }}$ pessoa correferencial e nãocorreferencial também seja relevante para verbos intransitivos que são núcleos de oração dependente em Wayoro (cf. exemplo (15) acima), nesta língua, quando o índice de pessoa é usado como argumento absolutivo sujeito de oração complemento infinitiva, há restrições nas possibilidades de uso do índice de $3^{\text {a }}$ pessoa correferencial, definidas conforme seleção do verbo da sentença matriz (cf. NOGUEIRA, 2019, p.118-125). Esta autora utiliza a diferença na possibilidade de uso do índice correferencial como recurso para identificação de orações infinitivas de controle. As orações infinitivas complementos do verbo toa 'ver' e as infinitivas complemento do verbo ndia 'querer' apresentam comportamento distinto quanto à possibilidade de uso do índice correferencial na posição de sujeito da encaixada, conforme exemplificado nos exemplos (16a-b) e (17a-c), a seguir.

Com o verbo ndia 'querer' na oração matriz, o sujeito da matriz controla o sujeito da encaixada. Neste caso, se o verbo da oração encaixada é intransitivo, os sujeitos das duas orações (matriz e subordinada) são correferenciais e o uso do índice de $3^{\mathrm{a}}$ pessoa correferencial te- é mandatório (16a). Desse modo, orações em que o sujeito da subordinada não é correferencial com o sujeito da matriz são agramaticais. A expressão de orações equivalentes a 'Djeopõri quer que eleleu tome banho', com sujeitos distintos entre a matriz e a encaixada, que se referem a uma disposição exterior que o sujeito exerce sobre outra pessoa, ou seja, o sujeito exerce controle sobre o objeto $^{14}$, apresentam outra estrutura morfossintática, em que se utiliza o verbo auxiliar mangã 'mandar, pedir', como em (16b).

(16) Wayoro

$\begin{array}{llll}\text { a. } & \text { Djeopõri } & \text { te-ato-a- } p & \text { ndia- } k w a-t \\ & \text { Djeopõri } & \text { 3C-banhar.se-V.T-INF } & \text { querer-ITER-NFUT }\end{array}$

'Djeopõri quer tomar banho.'
b. Djeopõri
$d j / m b=$ ato-ro-mang $\tilde{a}$
mbopoe
emõ

\footnotetext{
${ }^{14}$ Agradecemos a um(a) parecerista anônimo/a referência a essa distinção semântica entre o verbo querer indicando uma disposição interior do sujeito da matriz e uma disposição exterior que o sujeito exerce sobre outra pessoa, que ocorre em determinadas línguas.
}

Revista Moara, n. 56, vol. 2, jan-jul 2021 ISSN: 0104-0944 
Djeopõri 3/1SG=banhar.se-?-AUX rápido ENF

'Djeopõri está mandando ele/eu tomar banho rápido mesmo.'

Situação oposta ocorre com o verbo toa 'ver' na oração matriz. Neste caso, o sujeito da matriz não controla o sujeito da encaixada. Desse modo, quando um verbo intransitivo ocorre como núcleo da encaixada, não é possível o uso do índice de pessoa correferencial, conforme ilustrado em (17b), mas ao contrário utiliza-se o índice de $3^{\mathrm{a}}$ pessoa não-correferencial, indicando que os sujeitos não são idênticos (17a). A expressão de sujeitos correferenciais entre a oração matriz e a subordinada é realizada com emprego de duas sentenças coordenadas (17c).

(17) Wayoro

\begin{tabular}{|c|c|c|c|c|c|}
\hline \multirow[t]{3}{*}{ a. } & Djeopõri & $d j=a t o-a-p$ & top- $a-t$ & & \\
\hline & Djeopõri & 3=banhar.se-V.T-INF & ver-V.T-NFUT & & \\
\hline & Djeopõri $_{i} \mathrm{v}$ & ele $_{* i j}$ tomando banho. & & & \\
\hline \multirow[t]{2}{*}{ b. } & *Djeopõrĩ & te-ato-a-p & top- $a-t$ & & \\
\hline & Djeopõri & 3=banhar.se-V.T-INF & ver-V.T-NFUT & & \\
\hline \multirow[t]{4}{*}{ c. } & Djeopõri & te-ato-a & kap-ere & $u g u$ & $p i=e r i$ \\
\hline & D. & 3C-banhar.se-V.T & PROFORMA-em & água & dentro $=\mathrm{ABL}$ \\
\hline & te-ndaop & top-a- $t$ & & & \\
\hline & 3C-reflexo & ver-V.T-NFUT & & & \\
\hline
\end{tabular}

\section{Sobre a origem do sistema de rastreamento de referência intercláusulas: índices de $3^{\mathrm{a}}$ pessoa correferencial e não-correferencial}

Como vimos nas seções anteriores, existe uma relação entre sistema de marcação de argumentos em sentenças simples nas línguas do ramo Tupari com o sistema de rastreamento e controle de referência em sentenças complexas, baseado especificamente na distinção entre duas formas do índice de $3^{\mathrm{a}}$ pessoa (correferencial e não-correferencial). Apresentamos aqui uma hipótese sobre o desenvolvimento dessa estratégia para rastreamento e controle de referência, como uma extensão do sistema de marcação de argumento encontrado em sentenças simples.

Revista Moara, n. 56, vol. 2, jan-jul 2021 ISSN: 0104-0944 
Como descrito na seção 1 , nas sentenças simples, o índice de $3^{\mathrm{a}}$ pessoa correferencial é usado como índice de sujeito com verbos intransitivos, enquanto o índice de $3^{\mathrm{a}}$ pessoa não-correferencial é utilizado como índice de objeto, com verbos transitivos, conforme exemplificado em (18a-b), para a língua Sakurabiat.

\section{Estágio 1:}

(18) Sakurabiat

a. améko $i_{O B J}$-So-a-t

cachorro 3SG-ver-V.T-PASS

'O cachorro viu ele/ela/isto.'
b. améko se $e_{S U B J}-k w e a r-a-t$
cachorro 3C-correr-V.T-PASS
'O cachorro correu.'

Tomando por base os parâmetros de codificação, ou seja, a estratégia formal para marcar identidade e diferença na identidade dos referentes dos argumentos (distinção entre os dois índices de $3^{\mathrm{a}}$ pessoa - correferencial e não-correferencial), locus da marcação (verbos), o domínio relevante para a interpretação da referência (local ou de longa distância) e a natureza sintática do controlador e do pivot nas relações de identidade/diferença das referências dos participantes, a estratégia de rastreamento de referência intercláusulas descrita na seção 2.2 pode ser derivada a partir de uma extensão do sistema de marcação de argumentos em sentenças simples.

Nesse sentido, teríamos inicialmente, o índice correferencial indexando o sujeito e o índice não-correferencial indexando o objeto (estágio 1), como mostrado em (18a-b) acima, em sentenças simples (domínio local). Por analogia, em um segundo estágio (estágio 2), em clásulas complexas, teríamos o índice de pessoa correferencial usado quando o sujeito de uma sentença é correferente com o sujeito de outra sentença e o índice não-correferencial usado quando o sujeito de uma sentença é correferente com o objeto de outra sentença, como indicado em (19a-b).

\section{Estágio 2:}

(19) Sakurabiat
a. $\quad$ améko
$\operatorname{aose}_{j}$
sogo- $a-t$
$s e_{i}$-aor-a-ra
cachorro/onça homem
morder-V.T-PASS 3C-sair-V.T-REP

$\operatorname{se}_{i}-\operatorname{ses} \mathrm{-}-\mathrm{a}$

Revista Moara, n. 56, vol. 2, jan-jul 2021 ISSN: 0104-0944 
3C-AUX.andar.IPRF-PASS

'O cachorro ${ }_{i}$ mordeu o homem , enquanto ele $_{i}$ ia saindo'

$\begin{array}{llll}\text { b. } & \begin{array}{lll}\text { améko } \\ \text { cachorro/onça homem }\end{array} & \text { aose } & s e_{j} \text {-aor-a-ra } \\ & \text { morder-V.T-PASS } & \text { 3C-sair-V.T-REP }\end{array}$

$i_{j}$-seso-a

3C-AUX.andar.IPRF-PASS

'O cachorro $_{i}$ mordeu o homem $_{j}$, enquanto ele ${ }_{j}$ ia saindo'

Essa estrutura pode, então, ser reinterpretada (estágio 3), como indicando correferência e não-correferência entre sujeitos. O índice correferencial mantém o uso com sujeitos correferentes, enquanto o índice não-correferencial é usado com sujeitos diferentes, independente de correferência com o objeto de outra cláusula. Assim, podemos derivar o uso de índice de pessoa não-correferencial mesmo com verbos intransitivos, o que é agramatical em sentenças simples, conforme indicado em (20) e (21).

\section{Estágio 3}

(20) Sakurabiat

\begin{tabular}{|c|c|c|c|c|}
\hline$s e_{j-i-a}$ & (pro) & te & $i k \tilde{a} w$ & $i_{k}-e r-a$ \\
\hline 3C-vir-V.T & (sujeito) & FOC & DEM & 3-dormir-V.T \\
\hline$i_{k}-t o-a$ & & \multicolumn{3}{|c|}{$p e=o-m \tilde{e}_{k}$} \\
\hline 3-AUX.deit & .IPFV-PASS & \multicolumn{3}{|c|}{$\mathrm{OBL}=1 \mathrm{SG}-\mathrm{marido}$} \\
\hline
\end{tabular}

(21) Wayoro

$\begin{array}{lll}\text { Djeopõri } & d j=a t o-a-p & \text { top- } a-t \\ \text { Djeopõri } & 3=\text { banhar.se-V.T-INF } & \text { ver-V.T-NFUT } \\ \text { 'Djeopõri }{ }_{i} \text { viu ele }{ }_{* i j} \text { tomando banho.' }\end{array}$

Em Sakurabiat essa extensão do sistema de marcação de argumentos é irrestrita, mas em Wayoro, parece sofrer restrições em sentenças subordinadas, dependendo da seleção do verbo da matrix (cf. Seção 2.2 acima).

Em suma, em sentenças simples (domínio local), o índice de $3^{\mathrm{a}}$ pessoa correferencial tem uma função anafórica indicando correferencialidade com um sujeito (conominalização de sujeitos intransitivos, objeto reflexivo e possuidor de objeto correferente com o sujeito) e o índice não-correferencial indexa objeto não reflexivo e o possuidor de objeto não reflexivo. Propomos a extensão desse sistema de marcação de 
argumentos, em sentenças simples, para indicar de modo mais amplo uma distinção entre sujeitos correferentes e não correferentes. Ainda que bastante preliminar, essa hipótese é compatível com os dados das línguas analisadas e ajuda a explicar a ocorrência de sentenças nas quais um verbo intransitivo ocorre com o índice de pessoa não-correferencial, o que seria, de outra maneira, excluído como agramatical pelo sistema de marcação de argumentos descrito na seção 1 .

\section{Conclusão}

As línguas da família Tupi exibem duas propriedades que estão diretamente relacionadas aos diferentes padrões de alinhamento morfossintático encontrados na família e às estratégias de identificação das relações gramaticais e rastreamento de referência. Essas propriedades são a ocorrência de duas séries de morfemas pessoais morfemas livres (pronomes) e presos (índices de pessoa) - e a existência de um contraste entre formas pronominais identificadas como correferenciais e formas pronominais não-correferenciais. Analisamos, neste artigo, as principais estratégias morfossintáticas do sistema de rastreamento e controle de referências, para as línguas Sakurabiat e Wayoro, do ramo Tupari da família linguística Tupi. A análise foi centrada na interação entre marcação de argumentos, indexação de pessoa e o uso da oposição entre índices de pessoa correferencial e não correferencial para o rastreamento de referências em sentenças complexas.

Esperamos que as discussões apresentadas neste artigo sirvam de motivação para mais estudos de ordem comparativa e tipológica funcional de outras línguas da família Tupi, em especial sobre esse traço saliente da morfossintaxe das línguas dessa família, que é o contraste entre duas séries de morfemas pessoais - correferenciais e nãocorreferenciais - que é empregado com nomes possessivos e verbos. Compreender a interação entre esse sistema de marcar correferencialidade, codificação de argumentos no domínio local e o rastreamento e controle de referência no nível do discurso é especialmente relevante para o avanço do conhecimento da sintaxe das línguas Tupi. Com o presente trabalho, damos um passo em direção à análise unificada sobre a estrutura interna desse sistema, nas línguas Tupi e sua relação com sistemas de 
rastreamento e controle de referência, encontrados nas línguas do mundo ${ }^{15}$.

Finalizamos prestando homenagem à linguista Lucy Seki que dedicou grande parte da sua trajetória acadêmica ao avanço do conhecimento das línguas Tupi, especialmente da língua Kamayurá, e entre seus inúmeros trabalhos sobre as línguas dos povos originários do Brasil, também enveredou pelo estudo da língua Tupari, uma das cinco línguas do ramo Tupari (Tupi). Seu comprometimento e dedicação no estudo das línguas Tupi continuam servindo de inspiração para nós e para muitos estudiosos dessas línguas.

\section{Agradecimentos}

Gostaríamos de agradecer aos povos Sakurabiat e Wayoro, pela parceria e acolhimento aos nossos trabalhos com suas respectivas línguas, em especial, in memorian, a D. Vicência Sakyrabiar, D. Luzia Sakyrabiar, Sr. Pedro Kampé Sakyrabiar, D. Marina Pakoreiru Djeoromitxi, falecidos em 2020 e 2021. Sua força e seus ensinamentos ficarão para sempre guardados em nossos corações. Galucio agradece à Bolsa de Produtividade em Pesquisa do CNPQ - Processo 305843/2019-2.

\section{Abreviações}

1SG primeira pessoa singular; 2SG segunda pessoa singular; 3SG terceira pessoa nãocorreferencial; 1PL.INCL primeira pessoa plural inclusivo; 1PL.EXCL primeira pessoa plural exclusivo; 3C terceira pessoa correferencial; ABL ablativo; AUX auxiliar; CAUS causativo; COP copula; C correferencial; DAT dativo; DEM demonstrativo; DESID desiderativo; DIM diminutivo; ENF ênfase; EVID evidencial; FOC foco; FUT futuro; IM.FUT futuro imediato; INF infinitivo; INTENS intensificador; INTRZVR intransivizador; IPFV imperfectivo; IRR irrealis; ITER iterativo; LOC locativo; MED medial; NASSERT nãoassertivo; NFUT não-futuro NEG negação; NMLZ nominalizador; NPASS não-passado; OBJ objeto direto; OBL oblíquo; P pessoa; PL plural; PL.EV plural de eventos; PFV perfectivo; PAS passado; REP repetitivo; RED duplicação; SG singular; SN sintagma nominal; SOC sociativo; SOV sujeito-objeto-verbo; SV sintagma verbal; SUBJ sujeito; vBLZ verbalizador; V.T vogal temática; VZR verbalizador; TOP tópico; TR transitivizador

\footnotetext{
${ }^{15}$ Este tópico é o objeto de outro artigo, em preparação.
} 


\section{REFERÊNCIAS}

ALVES, P. M. O léxico do Tuparí: proposta de um dicionario bilíngue. Tese (Doutorado em Linguística e Língua Portuguesa) - Faculdade de Ciências e Letras, Universidade Estadual Paulista 'Júlio de Mesquita', 2004.

ARAGON, C. C. A Grammar of Akuntsú: a tupían Language. Tese (Doutorado) University of Hawai'i at Manoa, Mānoa, 2014.

ASUDEH, A.; DALRYMPLE, M. Binding theory. In: BROWN, K. (Ed.) Encyclopedia of language and linguistics. Amsterdam: Elsevier, 2006. v. 2. p. 23-31.

BIRCHALL, J. Argument marking patterns in South American languages. Utrecht: Netherlands Graduate School of Linguistics, 2014.

CHOMSKY, N. Lectures on Government and Binding. Dordrecht: Foris, 1981.

COMRIE, B.; HASPELMATH, M.; BICKEL, B. (Eds.). The Leipzig glossing rules: Conventions for interlinear morpheme-by-morpheme glosses. Leipzig: Department of Linguistics of the Max Planck Institute for Evolutionary Anthropology and of the University of Leipzig, 2008. Atualizado em 2015. Disponível em: http://www.eva.mpg.de/lingua/resources/glossing-rules.php, Acesso em: 25/03/2021.

GALUCIO, A. V. Argument marking and reference tracking in Mekens. In: GIJN, R. van; HAMMOND, J.; MATIC, D.; PUTTEN, S. van; GALUCIO, A. V. (Ed.). Information structure and reference tracking in complex sentences. Amsterdam/Philadelphia: John Benjamins, 2014. p. 373-396.

GALUCIO, A. V.; NOGUEIRA, A. F. de S. From object nominalization to object focus. Journal of Historical Linguistics, Amsterdam/Philadelphia, v. 8, n. 1, p. 95-127, 2018.

GALUCIO, V.; MOORE, D.; VOORT, H. V. D. O patrimônio linguístico do Brasil: novas perspectivas e abordagens no planejamento e gestão de uma política da diversidade linguística. Revista do Patrimônio Histórico e Artístico Nacional, v. 38, p. 195-221, 2018.

GILDEA, S.; CASTRO ALVES, F. Nominative-Absolutive: Counter-Universal Split Ergativity in Jê and Cariban. In: GILDEA, S.; QUEIXALÓS, F. Ergativity in Amazonia. Typological Studies in Language. Amsterdam: John Benjamins, 2010. p. 159-199.

Revista Moara, n. 56, vol. 2, jan-jul 2021 ISSN: 0104-0944 
GILDEA, S.; CASTRO ALVES, F. Reconstructing the Source of NominativeAbsolutive Alignment in Two Amazonian Language Families. In: LUJÁN, E.; BARĐDAL, J.; GILDEA, S. (Orgs.). Reconstructing Syntax: Cognates and Directionality. Leiden: Brill Press, 2018.

HASPELMATH, M. On S, A, P, T, and R as comparative concepts for alignment typology. Linguistic Typology, Berlin, v. 15, n. 3, p. 535-567, 2011. doi.org/10.1515/LITY.2011.035

HASPELMATH, M. Argument indexing: A conceptual framework for the syntactic status of bound person forms. In: BAKKER, D.; HASPELMATH, M. (Eds). Languages across boundaries. Berlin: De Gruyter Mouton, 2013. p. 197-226. doi.org/10.1515/9783110331127

JENSEN, C. Comparative Tupi-Guarani Morphosyntax. In: DERBYSHIRE, D. C.; PULlUM, G. K. (Eds). Handbook of Amazonian Languages. Berlin/New York: Mouton de Gruyter, 1998. v. 4. p. 491-618.

MATIĆ, D.; VAN GIJN, R.; VAN VALIN JR, R. D. Information structure and reference tracking in complex sentences: an overview. In: VAN GIJN, R.; HAMMOND, J.; MATIĆ, D.; VAN PUTTEN, S; GALUCIO, A. V. Information structure and reference tracking in complex sentences. Amsterdam/Philadelphia: John Benjamins, 2014.

NOGUEIRA, Antônia Fernanda de Souza. Predicação na língua Wayoro (Tupi): propriedades de finitude. 2019. 189 f. Tese (Doutorado em Semiótica e Linguística Geral) - Faculdade de Filosofia, Letras e Ciências Humanas da Universidade de São Paulo, São Paulo, 2019.

NOGUEIRA, Antônia Fernanda de Souza. Wayoro ẽmẽto: fonologia segmental e morfossintaxe verbal. 2011. 238 f. Dissertação (Mestrado em Semiótica e Linguística Geral) - Faculdade de Filosofia, Letras e Ciências Humanas da Universidade de São Paulo, São Paulo, 2011.

RODRIGUES, A.; CABRAL, A. S. A. C. Tupian. In: CAMPBELL, L.; GRONDONA, V. (Eds.). The indigenous languages of South America: a comprehensive guide. Berlin/Boston: De Gruyter Mouton, 2012. p. 495-574.

SHOPEN, T. (Ed.). Language typology and syntactic description. Cambridge: Cambridge University Press. v. 1, 2, 3. 
SINGERMAN, A. R. The morphosyntax of Tuparí, a Tupían language of the Brazilian Amazon. Tese (Doutorado) - Departamento de Linguística, Universidade de Chicago, Chigado, 2018.

STORTO, L. R. Línguas Indígenas: Tradição, Universais e Diversidade. Campinas: Mercado de Letras, 2019. v. 1. 194p.

VAN DER VOORT, H. A grammar of Kwaza. Berlin/New York: Mouton de Gruyter, 2004.

Revista Moara, n. 56, vol. 2, jan-jul 2021 ISSN: 0104-0944 